\title{
Hadron-quark hybrid model reliable for equations of state with $\mu_{B} \leq 400 \mathrm{MeV}$
}

\author{
Akihisa Miyahara, ${ }^{1}$ Masahiro Ishii, ${ }^{2}$ Hiroaki Kouno, ${ }^{3}$ and Masanobu Yahiro $\oplus^{2, *}$ \\ ${ }^{1}$ Observation Division, Chubu Aviation Weather Service Center, Japan Meteorological Agency, \\ Tokoname 479-0881, Japan \\ ${ }^{2}$ Department of Physics, Graduate School of Sciences, Kyushu University, Fukuoka 819-0395, Japan \\ ${ }^{3}$ Department of Physics, Saga University, Saga 840-8502, Japan
}

(Received 24 July 2019; accepted 23 March 2020; published 13 April 2020)

\begin{abstract}
We present a simple version of hadron-quark hybrid $(\mathrm{HQH})$ model in the $\mu_{B}-T$ plain, where $T$ is temperature and $\mu_{B}$ is the baryon-number chemical potential. The model is composed of the independentquark model for quark-gluon states and an improved version of excluded-volume hadron resonance gas (EV-HRG) model for hadronic states. In the improved version of EV-HRG, the pressure has charge conjugation and is obtained by a simple analytic form. The switching function from hadron states to quarkgluon states in the present model has no chemical potential dependence. The simple HQH model is successful in reproducing LQCD results on the transition region of chiral crossover and the EoS in $\mu_{B} \leq 400 \mathrm{MeV}$. We then predict the chiral-crossover region in $400 \leq \mu_{B} \leq 800 \mathrm{MeV}$. We also predict a transition line derived from isentropic trajectories in $0 \leq \mu_{B} \leq 800 \mathrm{MeV}$ and find that the effect of strangeness neutrality is small there.
\end{abstract}

DOI: $10.1103 /$ PhysRevD.101.076011

\section{INTRODUCTION}

\section{A. LQCD}

The state-of-art $2+1$-flavor lattice QCD (LQCD) simulation of Ref. [1] showed that the transition is "crossover" at finite temperature $(T)$ and zero baryon chemical potential $\left(\mu_{B}=0\right)$, where the continuum and thermodynamic limits were carefully taken. In general, the crossover nature means that the transition temperature depends on the choice of observables. In fact, observable-dependent transition temperatures $T_{c}^{(O)}\left(\mu_{B}\right)$ have been discussed in LQCD simulations for zero and small $\mu_{B}$; actually, the renormalized chiral condensate $O=\Delta_{l, s}\left(T, \mu_{B}\right)$, the Polykov loop $O=\Phi\left(T, \mu_{B}\right)$, the energy density $O=\varepsilon\left(T, \mu_{B}\right)$, and the trace anomaly $O=I\left(T, \mu_{B}\right)$ are taken in Refs. [2-9]. In Ref. [9], the LQCD data disfavor the existence of critical endpoint (CEP) in $\mu_{B} / T \leq 2$ and $T / T_{c}^{\left(\Delta_{l, s}\right)}\left(\mu_{B}=0\right)>0.9$. The equation of state $(\mathrm{EoS})$ is important particularly for relativistic nuclear collisions and neutron stars. The location of transition region is essential to determine EoS. For

*orion093g@gmail.com

Published by the American Physical Society under the terms of the Creative Commons Attribution 4.0 International license. Further distribution of this work must maintain attribution to the author(s) and the published article's title, journal citation, and DOI. Funded by SCOAP ${ }^{3}$. these reasons, a lot of LQCD data have been accumulated [1-13].

\section{B. Effective models}

As a complementary approach to LQCD simulations, we can consider effective models such as the quark-meson model [14] and the Polyakov-loop extended Nambu-JonaLasinio (PNJL) model [15-18]. The model approach is useful for the prediction of the transition lines, the presence or absence of CEP and EoS. The hadron resonance gas (HRG) model is a simple model for hadronic matter and remarkably reproduces LQCD data on $\mathrm{EoS}$ in $T \lesssim$ $1.3 T_{c}^{\left(\Delta_{l, s}\right)}\left(\mu_{B}=0\right)[11]$.

As a simplified version of the PNJL model [15-18], the independent-quark (IQ) model reproduces $T$ dependence of the Polyakov loop calculated with 2+1-flavor LQCD simulations for $\mu_{B}=0[19,20]$, although the PNJL model does not. The IQ model treats the coupling between the quark field and the homogeneous classical gauge field, but not the couplings between quarks.

\section{Hadron-quark hybrid model}

Asakawa and Hatsuda proposed the hadron-quark hybrid $(\mathrm{HQH})$ model for $\mu_{B}=0$ in order to describe the coexistence of quarks and hadrons [21]. The total entropy $s\left(T, \mu_{B}\right)$ of the model is $s(T)=f_{\mathrm{H}}(T) s_{\mathrm{H}}(T)+\left[1-f_{\mathrm{H}}(T)\right] s_{\mathrm{Q}}(T)$, where $s_{\mathrm{H}}(T) \equiv 12\left(\pi^{2} / 90\right) T^{3}$ and $s_{\mathrm{Q}}(T) \equiv 148\left(\pi^{2} / 90\right) T^{3}$ are the entropy densities of massless free gas with two 


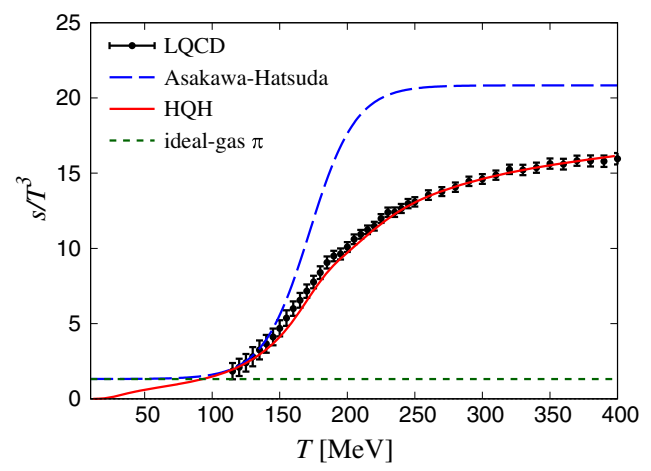

FIG. 1. $T$ dependence of $s / T^{3}$ at $\mu_{B}=0 \mathrm{MeV}$. The dashed line denotes the $s / T^{3}$ of Ref. [21] in which $s_{\mathrm{Q}}(T) / T^{3} \equiv 190\left(\pi^{2} / 90\right)$ for three-flavor free quark-gluon gas and $T_{c}=172 \mathrm{MeV}$, where $T_{c}$ is the chiral pseudocritical temperature at $\mu_{B}=0 \mathrm{MeV}$. The solid line stands for the result of Ref. [20]. The dotted line is $s_{\mathrm{H}}(T) / T^{3} \equiv 12\left(\pi^{2} / 90\right)$ of massless pion gas. LQCD data for $2+1$ flavor are taken from Ref. [7].

flavors in the hadronic phase (pion gas) and in the quarkgluon phase, respectively. The weight function $f_{\mathrm{H}}(T)$ means the occupancy of hadronic matter in the total entropy and assumed a simple function satisfying the condition $0 \leq f_{\mathrm{H}} \leq 1$. As shown in Fig. 1, their $s(T)$ (dashed line) does not reproduce $s_{\mathrm{LQCD}}$, where the $f_{\mathrm{H}}(T)$ has a width parameter $\Gamma / T_{c}$ and the value 0.2 has been determined to reproduce the low $T$ part of $s_{\mathrm{LQCD}}(T)$. In addition, their $s(T) / T^{3}$ does not vanish at $T=0$, but HRG does. Therefore, we should take HRG as $s_{\mathrm{H}}(T)$ and the IQ model as $s_{\mathrm{Q}}(T)$.

In our previous papers $[19,20]$, we improved the $\mathrm{HQH}$ model of Ref. [21] for finite $\mu_{B}$, taking the HRG model for the hadronic part and the IQ model for the quark-gluon part. The total entropy $s\left(T, \mu_{B}\right)$ reads

$$
s\left(T, \mu_{B}\right)=f_{\mathrm{H}}\left(T, \mu_{B}\right) s_{\mathrm{H}}\left(T, \mu_{B}\right)+\left[1-f_{\mathrm{H}}\left(T, \mu_{B}\right)\right] s_{\mathrm{Q}}\left(T, \mu_{B}\right) .
$$

The result (solid line) of Ref. [20] reproduces LQCD data [7], as shown in Fig. 1.

Another type of $\mathrm{HQH}$ model was proposed in Refs. [22,23]. The HQH model considers the pressure instead of the entropy. As an advantage of our approach, $s_{\text {LQCD }}$ automatically satisfies the thermodynamic inequality and the Nernst's theorem [24],

$$
\left.\frac{\partial s\left(T, \mu_{B}\right)}{\partial T}\right|_{\mu_{B}=0}>0,\left.\quad s\left(T, \mu_{B}\right)\right|_{T=\mu_{B}=0}=0 .
$$

In our previous papers $[19,20]$, the $f_{\mathrm{H}}\left(T, \mu_{B}\right)$ was determined from LQCD data on $s_{\mathrm{LQCD}}$ and the secondorder susceptibilities at $\mu_{B}=0$. For this reason, the approach is applicable only for small $\mu_{B}$. We could not show the chiral-transition line, since $\Delta_{1, \mathrm{~s}}$ becomes negative in $T \gtrsim 170 \mathrm{MeV}$.

In the HRG model, the interactions between baryons (antibaryon) are neglected, but it should be taken into account for $\mu_{B}$ dependence of thermodynamic quantities. A simple way of treating volume-exclusion effects (repulsive force) [25] was suggested in Refs. [26,27]. This model is called "excluded-volume HRG (EV-HRG) model." Furthermore, a method of treating an attractive force in addition to the repulsive force was proposed in Ref. [28]. The volume-exclusion effects are included by fitting the volume parameter $b=4 \cdot 4 \pi r^{3} / 3$ [24] to either LQCD data or the core radius $r$ of nucleon-nucleon force [26,27]. In the framework of Refs. [26-28], the interaction between baryon and antibaryon and the radius of meson are neglected.

\section{Our aim}

In this paper, we improve the HQH model of Ref. [20], taking the EV-HRG model for the hadron piece and the simple IQ model for the quark-gluon piece. The EV-HRG model taken yields the pressure as a simple analytic function and guarantees that the pressure is $\mu_{B}$ even. We refer to the present version of $\mathrm{HQH}$ model as "simple $\mathrm{HQH}$ (sHQH) model."

The present $\mathrm{sHQH}$ model has only six parameters, i.e., one parameter $r$ in the EV-HRG model and five parameters in the IQ model. In the IQ model, the parameters are fitted to $s_{\mathrm{LQCD}}$ in $400<T<800 \mathrm{MeV}$ and $\mu_{B}=0$ [20]. In our EV-HRG model, as a value of $r$, we take the hard-core radius $r=0.34 \mathrm{fm}$ of the Hamada-Johnston nucleonnucleon interaction [29], since the other nuclear forces do not have the hard core. We have also supposed that the hard core universally emerges in the other baryon-baryon interactions between hyperons or excited baryons, and their core radii are assumed to be the same as that of nucleon. We then determined the switching function $f_{\mathrm{H}}$ from $s_{\mathrm{LQCD}}$ at $\mu_{B}=0$. The sHQH model with the $f_{\mathrm{H}}(T, 0)$ reproduces LQCD data on the Polyakov loop at zero chemical potential and the EoS in finite $\mu_{B}$ up to $400 \mathrm{MeV}$. The present sHQH model thus has no $\mu_{B}$ in $f_{\mathrm{H}}$; namely, $\mu_{B}$ dependence of physical quantities come from the EV-HRG and the IQ model. We thus succeed in simplifying the HQH model by taking $r=0.34 \mathrm{fm}$.

The $\Delta_{1, \mathrm{~s}}$ signals the chiral transition. The $\Delta_{1, \mathrm{~s}}$ calculated with the HRG model becomes negative in $T \gtrsim 170 \mathrm{MeV}$ [5], whereas the corresponding LQCD result is positive. The present model has this problem. We circumvent this problem in the following way.

As an interesting result of LQCD simulations in Ref. [5], the chiral-crossover region determined from $d \Delta_{1, \mathrm{~s}} / d T$ agrees with that from $d \varepsilon / d T$ at $\mu_{B}=0$. In LQCD simulations of Ref. [7], furthermore, the transition region is obtained by $d \varepsilon / d T$ for finite $\mu_{B}$. Therefore, we use the peak and the half-value width of $d \varepsilon / d T$ as a transition region in $\mu_{B}-T$ plane. We show that the transition region determined 
from $\varepsilon$ agrees with the chiral-transition region calculated with LQCD simulations [8].

As mentioned above, the present sHQH model well reproduces LQCD data on the EoS and the chiral-crossover region in $0 \leq \mu_{B} \leq 400 \mathrm{MeV}$. We can then predict the transition region of chiral crossover in $400 \leq \mu_{B} \leq$ $800 \mathrm{MeV}$. LQCD data will become available for $\mu_{B}=$ $400 \sim 800 \mathrm{MeV}$ by development of LQCD simulations such as the complex Langevin method [30-33].

Finally, we present a transition line derived from isentropic trajectories in $0 \leq \mu_{B} \leq 800 \mathrm{MeV}$. When we calculate the isentropic trajectories, we switch on and off the strangeness neutrality. We find that the effect is small there. For this reason, we do not consider the strangeness neutrality for the chiral-crossover region and the EoS.

This paper is organized as follows. In Sec. II, we show the model building. Numerical results are shown in Sec III. Section IV is devoted to a summary.

\section{MODEL BUILDING}

We present a simple version of the $\mathrm{HQH}$ model. The model is composed of an improved version of EV-HRG model for hadronic states and the IQ model for quark-gluon states.

For the $2+1$ flavor system, we can consider the chemical potentials of $\mathrm{u}, \mathrm{d}, \mathrm{s}$ quarks by $\mu_{\mathrm{u}}, \mu_{\mathrm{d}}, \mu_{\mathrm{s}}$, respectively. These potentials are related to the baryon-number $(B)$ chemical potential $\mu_{B}$, the isospin $(I)$ chemical potential $\mu_{I}$, and the hypercharge $(Y)$ chemical potential $\mu_{Y}$ as

$$
\begin{aligned}
\mu_{B} & =\mu_{\mathrm{u}}+\mu_{\mathrm{d}}+\mu_{\mathrm{s}}, \\
\mu_{I} & =\mu_{\mathrm{u}}-\mu_{\mathrm{d}}, \\
\mu_{Y} & =\frac{1}{2}\left(\mu_{\mathrm{u}}+\mu_{\mathrm{d}}-2 \mu_{\mathrm{s}}\right) .
\end{aligned}
$$

As for $\mu_{I}$ and $\mu_{Y}$, the right-hand side of Eq. (3) comes from the diagonal elements of the matrix representation of Cartan algebra in $S U(3)$ group: $\mu_{I}=(1,-1,0)\left(\mu_{\mathrm{u}}, \mu_{\mathrm{d}}, \mu_{\mathrm{s}}\right)^{\mathrm{t}}$ and $\mu_{Y}=(1 / 2)(1,1,-2)\left(\mu_{\mathrm{u}}, \mu_{\mathrm{d}}, \mu_{\mathrm{s}}\right)^{\mathrm{t}}$. Equation (3) yields

$$
\begin{aligned}
& \mu_{\mathrm{u}}=\frac{1}{3} \mu_{B}+\frac{1}{2} \mu_{I}+\frac{1}{3} \mu_{Y}, \\
& \mu_{\mathrm{d}}=\frac{1}{3} \mu_{B}-\frac{1}{2} \mu_{I}+\frac{1}{3} \mu_{Y}, \\
& \mu_{\mathrm{s}}=\frac{1}{3} \mu_{B}-\frac{2}{3} \mu_{Y} .
\end{aligned}
$$

\section{A. HRG model}

For later convenience, we start with the HRG model. In the model, the pressure $P_{\mathrm{H}}$ is divided into the baryon (B) part $P_{\mathrm{B}}$, the antibaryon $(\mathrm{aB})$ part $P_{\mathrm{aB}}$, and the meson (M) part $P_{\mathrm{M}}$,

$$
P_{\mathrm{H}} \equiv P_{\mathrm{B}}+P_{\mathrm{aB}}+P_{\mathrm{M}},
$$

with

$$
\begin{gathered}
P_{\mathrm{B}}=\sum_{\mathrm{i} \in \mathrm{B}} d_{i} T \int \log \left(1+e^{-\left(E_{\mathrm{B}, i}-\mu_{\mathrm{B}, i}\right) / T}\right), \\
P_{\mathrm{aB}}=\sum_{\mathrm{i} \in \mathrm{aB}} d_{i} T \int \log \left(1+e^{-\left(E_{\mathrm{B}, i}+\mu_{\mathrm{B}, i}\right) / T}\right), \\
P_{\mathrm{M}}=-\sum_{\mathrm{j} \in \operatorname{Meson}} d_{j} T \int\left\{\log \left(1-e^{-\left(E_{\mathrm{M}, \mathrm{j}}-\mu_{\mathrm{M}, j}\right) / T}\right)\right. \\
\left.+\log \left(1-e^{-\left(E_{\mathrm{M}, j}+\mu_{\mathrm{M}, j}\right) / T}\right)\right\}
\end{gathered}
$$

for $E_{\mathrm{B}, i}=\sqrt{\mathbf{p}^{2}+m_{\mathrm{B}, i}{ }^{2}}$ and $E_{\mathrm{M}, j}=\sqrt{\mathbf{p}^{2}+m_{\mathrm{M}, j}{ }^{2}}$, where $m_{\mathrm{B}, i}\left(m_{\mathrm{M}, j}\right)$ and $\mu_{\mathrm{B}, i}\left(\mu_{\mathrm{M}, j}\right)$ are the mass and the chemical potential of the $i$ th baryon ( $j$ th meson), respectively. Here, we have used the shorthand notation

$$
\int \equiv \int \frac{d^{3} \mathbf{p}}{(2 \pi)^{3}}
$$

for the integration over 3d-momentum p. In Eq. (5), all the hadrons listed in the Particle Data Table [34] are taken.

\section{B. Improved version of EV-HRG}

We first explain the EV-HRG model of Refs. [26-28]. The pressure $P_{\mathrm{EV} ; \mathrm{H}}$ is obtained by

$$
\mathrm{P}_{\mathrm{EV} ; \mathrm{H}}=P_{\mathrm{EV} ; \mathrm{B}}+P_{\mathrm{EV} ; \mathrm{aB}}+P_{\mathrm{M}},
$$

with

$$
\begin{array}{r}
P_{\mathrm{EV} ; \mathrm{B}}=\sum_{\mathrm{i} \in \mathrm{B}} d_{i} T \int \log \left(1+e^{-\left(E_{\mathrm{B}, i}-\mu_{\mathrm{EV}: \mathrm{B}, i}\right) / T}\right), \\
P_{\mathrm{EV} ; \mathrm{aB}}=\sum_{\mathrm{i} \in \mathrm{aB}} d_{i} T \int \log \left(1+e^{-\left(E_{\mathrm{B}, i}+\mu_{\mathrm{EV}: \mathrm{aB}, i}\right) / T}\right) .
\end{array}
$$

Here the effective baryon and antibaryon chemical potentials, $\mu_{\mathrm{EV}: \mathrm{B}, i}$ and $\mu_{\mathrm{EV}: \mathrm{aB}, i}$, are defined by

$$
\begin{gathered}
\mu_{\mathrm{EV}: \mathrm{B}, i} / T=\mu_{B, i} / T-\bar{b} P_{\mathrm{EV} ; \mathrm{B}} / T^{4}, \\
\mu_{\mathrm{EV}: \mathrm{aB}, i} / T=\mu_{B, i} / T-\bar{b} P_{\mathrm{EV} ; \mathrm{aB}} / T^{4},
\end{gathered}
$$

where $\bar{b}=b T^{3}$ for a positive volume parameter $b$. It is not easy to obtain $P_{\mathrm{EV} ; \mathrm{B}}$ and $P_{\mathrm{EV} ; \mathrm{aB}}$, since $\mu_{\mathrm{EV} ; \mathrm{B}, i}\left(\mu_{\mathrm{EV} ; \mathrm{aB}, i}\right)$ includes $P_{\mathrm{EV} ; \mathrm{B}}\left(P_{\mathrm{EV} ; \mathrm{aB}}\right)$ and self-consistent calculation is necessary. Actually, $P_{\mathrm{EV} ; \mathrm{B}}$ and $P_{\mathrm{EV} ; \mathrm{aB}}$ are obtained by solving Eqs. (11) and (12) numerically. 
In $\mathrm{QCD}$, the pressure is charge-conjugation even $\left(\mu_{B}\right.$ even). Hence, $P_{\mathrm{EV} ; \mathrm{H}}$ should be $\mu_{B}$ even, because it is a model of explaining QCD in $T<T_{c}$. Since $\mu_{\mathrm{EV}: \mathrm{B}, i}$ includes a $\mu_{B^{-}}$ odd term, $\mu_{B, i} / T$ and a $\mu_{B}$-even $\bar{b} P_{\mathrm{EV} ; \mathrm{B}} / T^{4}$, the resulting $P_{\mathrm{EV} ; \mathrm{H}}$ is not $\mu_{B}$ even. It is not natural.

We then redefine $P_{\mathrm{EV} ; \mathrm{B}}$ and $P_{\mathrm{EV} ; \mathrm{aB}}$ so that $P_{\mathrm{EV} ; \mathrm{H}}$ can be $\mu_{B}$ even. The redefined $P_{\mathrm{EV} ; \mathrm{B}}$ and $P_{\mathrm{EV} ; \mathrm{aB}}$ are denoted by $P_{\text {inv;B }}$ and $P_{\text {inv;aB }}$, respectively. Namely,

$$
\begin{array}{r}
P_{\mathrm{inv} ; \mathrm{B}}=\sum_{\mathrm{i} \in \mathrm{B}} d_{i} T \int \log \left(1+e^{-\left(E_{\mathrm{B}, i}-\mu_{\mathrm{inv} ; \mathrm{B}, i}\right) / T}\right), \\
P_{\mathrm{inv} ; \mathrm{aB}}=\sum_{\mathrm{i} \in \mathrm{aB}} d_{i} T \int \log \left(1+e^{-\left(E_{\mathrm{B}, i}+\mu_{\mathrm{inv} ; \mathrm{aB}, i}\right) / T}\right),
\end{array}
$$

with

$$
\begin{gathered}
\mu_{\text {inv }: \mathrm{B}, i} / T=\mu_{B, i} / T-\bar{b} P_{\mathrm{inv} ; \mathrm{B}} / T^{4}, \\
\mu_{\text {inv }: \mathrm{aB}, i} / T=\mu_{B, i} / T+\bar{b} P_{\text {inv;aB }} / T^{4} .
\end{gathered}
$$

The sum of $P_{\text {inv; B }}$ and $P_{\text {inv;aB }}$ are $\mu_{B}$ even, since the sum is invariant under $\mu_{B} \rightarrow-\mu_{B}$. For this reason, we take Eqs. (15)-(18). These equations show that $P_{\text {inv;B }} \geq P_{\text {inv;aB }}$.

$P_{\text {inv:B }}$ and $P_{\text {inv:aB }}$ can be rewritten into

$$
\begin{aligned}
\frac{P_{\mathrm{inv}: \mathrm{B}}}{T^{4}}= & \sum_{\mathrm{i} \in \mathrm{B}} A_{i} \sum_{\ell=1}^{\infty} \frac{(-1)^{\ell+1}}{\ell^{2}} \\
& \times K_{2}\left(\frac{\ell m_{i}}{T}\right) \exp \left(\frac{\ell \mu_{\mathrm{inv} ; \mathrm{B}, i}}{T}\right), \\
\frac{P_{\mathrm{inv}: \mathrm{aB}}}{T^{4}}= & \sum_{\mathrm{i} \in \mathrm{aB}} A_{i} \sum_{\ell=1}^{\infty} \frac{(-1)^{\ell+1}}{\ell^{2}} \\
& \times K_{2}\left(\frac{\ell m_{i}}{T}\right) \exp \left(-\frac{\ell \mu_{\mathrm{inv} ; \mathrm{aB}, i}}{T}\right)
\end{aligned}
$$

for

$$
A_{i} \equiv \frac{d_{i}}{2 \pi^{2}}\left(\frac{m_{i}}{T}\right)^{2}
$$

LQCD data on the EoS are available for $T \leq 400 \mathrm{MeV}$ and $\mu_{B} \leq 400 \mathrm{MeV}[5,7]$. We then consider this region. We consider $P_{\mathrm{B}}$, because of $P_{\text {inv; B }} \geq P_{\text {inv;aB }}$. The $\ell$ convergence of Eq. (19) becomes worse as $\left|\left(\mu_{B}-m_{i}\right) / T\right|$ becomes larger; note that $K_{2}(x)$ is proportional to $\exp (-x)$ for large $x$ and $\mu_{B}-m_{i}$ is negative. Therefore, the convergence is worst for the smallest case $(939-400) / 400$ where $T=\mu_{B}=$ $400 \mathrm{MeV}$ and $m_{N}=939 \mathrm{MeV}$. Taking the $\ell=1$ term only is a $3 \%$ error in Eq. (19). In actual calculations, nucleon contribution in $P_{\mathrm{B}}$ is only $3 \%$, so that taking the $\ell=1$ term only corresponds to $0.1 \%$ error. We can identify $P_{\mathrm{B}}$ with its $\ell=1$ term and $P_{\mathrm{aB}}$ with its $\ell=1$ one. This approximation is called " $\ell=1$ identification" in this paper.

Using the $\ell=1$ identification, we can rewrite $P_{\text {inv:B }}$ as

$$
\frac{P_{\text {inv }: \mathrm{B}}}{T^{4}} \simeq \sum_{\mathrm{i} \in \mathrm{B}} A_{i} K_{2}\left(\frac{m_{i}}{T}\right) \exp \left(\frac{\mu_{\mathrm{inv} ; \mathrm{B}, i}}{T}\right) .
$$

Multiplying both the sides of Eq. (22) by $\bar{b} \exp \left(\bar{b} P_{\text {inv; }} / T^{4}\right)$ and using the $\ell=1$ identification, one can obtain

$$
\begin{aligned}
\bar{b} & \frac{P_{\text {inv }: \mathrm{B}}}{T^{4}} \exp \left(\bar{b} \frac{P_{\text {inv; }}}{T^{4}}\right) \\
& =\bar{b} \sum_{\mathrm{i} \in \mathrm{B}} A_{i} K_{2}\left(\frac{m_{i}}{T}\right) \exp \left(\frac{\mu_{B, i}}{T}\right) \\
& =\bar{b} \sum_{\mathrm{i} \in \mathrm{B}} A_{i} \sum_{\ell=1}^{\infty} \frac{(-1)^{\ell+1}}{\ell^{2}} K_{2}\left(\frac{\ell m_{i}}{T}\right) \exp \left(\frac{\ell \mu_{B, i}}{T}\right) \\
& =\bar{b} \frac{P_{\text {inv } ;}\left(\mu_{B}\right)}{T^{4}}, \quad \text { for } \mu_{B}=\mu_{B, i} .
\end{aligned}
$$

Noting that the Lambert $W(z)$ function is the inverse function of $W e^{W}=z$, one can get $P_{\text {inv:B }}$ as a simple analytic function. Namely,

$$
\frac{P_{\text {inv:B }}}{T^{4}}=\frac{W\left(\bar{b} P_{\mathrm{inv} ; \mathrm{B}}\left(\mu_{B}\right) / T^{4}\right)}{\bar{b}} .
$$

In the limit of $\bar{b}=0, P_{\text {inv:B }}$ tends to $P_{\mathrm{B}}$, because of $W(z) \rightarrow z$. Parallel discussion is possible for antibaryon. The result is

$$
\frac{P_{\mathrm{inv} ; \mathrm{aB}}}{T^{4}}=\frac{W\left(\bar{b} P_{\mathrm{aB}}\left(\mu_{B}\right) / T^{4}\right)}{\bar{b}} .
$$

Hence, the hadronic pressure becomes

$$
\mathrm{P}_{\mathrm{inv} ; \mathrm{H}}=P_{\mathrm{inv} ; \mathrm{B}}+P_{\mathrm{inv} ; \mathrm{aB}}+P_{\mathrm{M}},
$$

with Eqs. (24) and (25). The entropy density $s_{\text {inv:H }}$ is obtained from $P_{\text {inv:H }}$ as

$$
s_{\text {inv }: \mathrm{H}}=\frac{\partial P_{\text {inv }: \mathrm{H}}}{\partial T} .
$$

This improved version of EV-HRG model is referred to as "improved EV-HRG model," but the difference between the improved EV-HRG model and the original EV-HRG model is not large for the pressure.

Figure 2 shows $T$ dependence of the total pressure $P$ for $\mu_{B}=0,400 \mathrm{MeV}$. The results of improved EV-HRG and HRG models are compared with LQCD ones [13]. In the improved EV-HRG model, we take the core radius $0.34 \mathrm{fm}$ as a value of $r$, i.e., $b=0.63 \mathrm{fm}^{3}$. For $\mu_{B}=400 \mathrm{MeV}$ (lower panel), the EV-HRG result (solid line) agrees with 

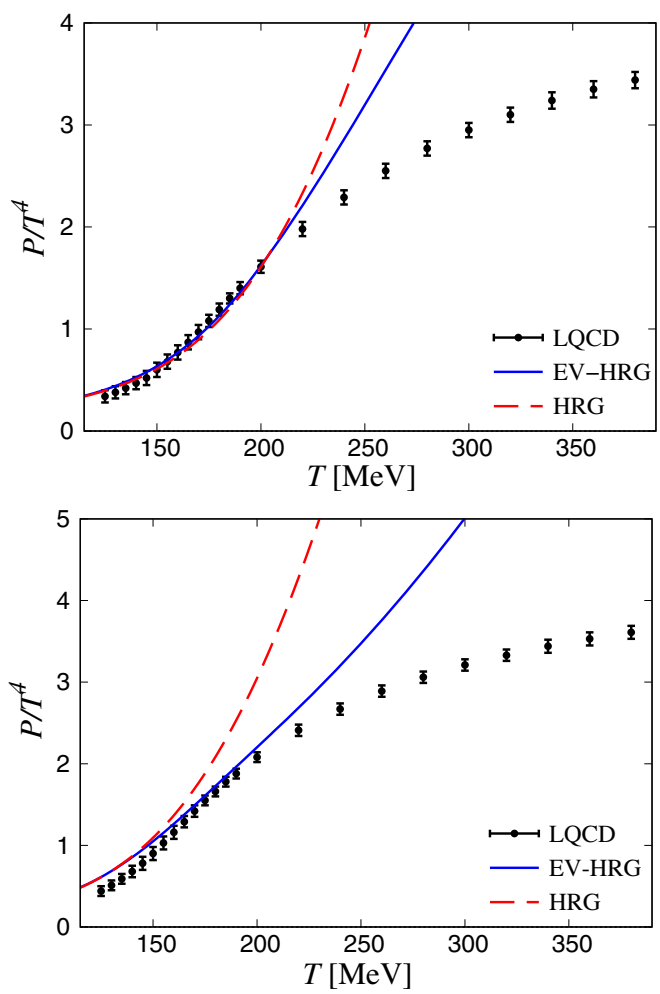

FIG. 2. $T$ dependence of pressure $P$ at $\mu_{B}=0 \mathrm{MeV}$ (upper panel) and $\mu_{B}=400 \mathrm{MeV}$ (lower panel). The solid and dashed lines stand for the results of improved EV-HRG model and HRG model, respectively. LQCD data are taken from Ref. [13].

LQCD one [13] in $T \lesssim 210 \mathrm{MeV}$, while the HRG result (dashed line) is consistent with LQCD one in $T \lesssim 150 \mathrm{MeV}$. For $\mu_{B}=0 \mathrm{MeV}$ (upper panel), both the EV-HRG and HRG results are consistent with LQCD one [13] in $T \lesssim 210 \mathrm{MeV}$. The difference between the EVHRG and HRG results means a repulsive nature of baryon and baryon.

\section{Independent-quark model}

We have to consider quark-gluon states in the region $T \gtrsim 200 \mathrm{MeV}$. The Lagrangian density of the IQ model is

$$
\mathcal{L}_{\mathrm{Q}}=\sum_{f}\left\{\bar{q}_{f}\left(i \gamma^{\mu} D_{\mu}-m_{f}\right) q_{f}\right\}-\mathcal{U}(T, \Phi, \bar{\Phi}),
$$

where $m_{f}$ is the current mass of $f$ quark and $D_{\mu}=\partial_{\mu}-$ $i g A_{\mu}^{a} \frac{\lambda_{a}}{2} \delta^{\mu 0}$ with the Gell-Mann matrix $\lambda_{a}$ in color space. See Refs. $[17,18]$ for the definition of the Polyakov loop $\Phi$ and its conjugate $\bar{\Phi}$.

Making the path integral over quark fields leads to

$$
P_{\mathrm{Q}}=-\mathcal{U}(T, \Phi, \bar{\Phi})+2 \sum_{f}\left[\int\left(T \log z_{f}^{+}+T \log z_{f}^{-}\right)\right] \text {, }
$$

where
TABLE I. Parameters in the Polyakov-loop potential.

$$
\begin{array}{lcccc}
\hline \hline a_{0} & a_{1} & a_{2} & b_{3} & T_{0} \\
\hline 2.457 & -2.47 & 15.2 & -1.75 & 270[\mathrm{MeV}] \\
\hline \hline & \\
z_{f}^{+}= & 1+3 \bar{\Phi} e^{-\left(E_{f}+\mu_{f}\right) / T}+3 \Phi e^{-2\left(E_{f}+\mu_{f}\right) / T} \\
& +e^{-3\left(E_{f}+\mu_{f}\right) / T}, \\
z_{f}^{-}= & 1+3 \Phi e^{-\left(E_{f}-\mu_{f}\right) / T}+3 \bar{\Phi} e^{-2\left(E_{f}-\mu_{f}\right) / T} \\
& +e^{-3\left(E_{f}-\mu_{f}\right) / T},
\end{array}
$$

with $E_{f}=\sqrt{\mathbf{p}^{2}+m_{f}^{2}}$. In Eq. (29), the vacuum term has been omitted, since the pressure calculated with LQCD simulations does not include the term. The $\Phi$ and $\bar{\Phi}$ are obtained by minimizing $\Omega_{\mathrm{Q}}=-P_{\mathrm{Q}}$.

The entropy density $s_{\mathrm{Q}}$ is obtained from $P_{\mathrm{Q}}$ as

$$
s_{\mathrm{Q}}=\frac{\partial P_{\mathrm{Q}}}{\partial T} .
$$

We take the Polyakov-loop potential of Ref. [20],

$$
\begin{gathered}
\frac{\mathcal{U}(T, \Phi, \bar{\Phi})}{T^{4}}=-\frac{a(T)}{2} \Phi \bar{\Phi} \\
+b(T) \log \left\{1-6 \Phi \bar{\Phi}+4\left(\Phi^{3}+\bar{\Phi}^{3}\right)\right. \\
\left.-3(\Phi \bar{\Phi})^{2}\right\}, \\
a(T)=a_{0}+a_{1}\left(\frac{T_{0}}{T}\right)+a_{2}\left(\frac{T_{0}}{T}\right)^{2}, \\
b(T)=b_{3}\left(\frac{T_{0}}{T}\right)^{3} .
\end{gathered}
$$

The parameters $a_{0}, a_{1}, a_{2}, b_{3}$, and $T_{0}$ were fitted to $2+1$ flavor $s_{\mathrm{LQCD}}$ in $400 \lesssim T \lesssim 500 \mathrm{MeV}$; see Fig. 1 of Ref. [20] for the fit. The resulting values are tabulated in Table I.

\section{D. sHQH model}

The total entropy reads

$$
s\left(T, \mu_{B}\right)=f_{\mathrm{H}}(T) s_{\text {inv }: \mathrm{H}}\left(T, \mu_{B}\right)+\left[1-f_{\mathrm{H}}(T)\right] s_{\mathrm{Q}}\left(T, \mu_{B}\right)
$$

in the sHQH model. We consider that $f_{\mathrm{H}}\left(T, \mu_{B}\right)$ has no $\mu_{B}$ dependence, since $s_{\text {inv: } \mathrm{H}}$ and $s_{\mathrm{Q}}$ depend on $\mu_{B}$. This allows us to determine $f_{\mathrm{H}}(T)$ from $s=s_{\mathrm{LQCD}}$ [13] at $\mu_{B}=0$. Namely, 


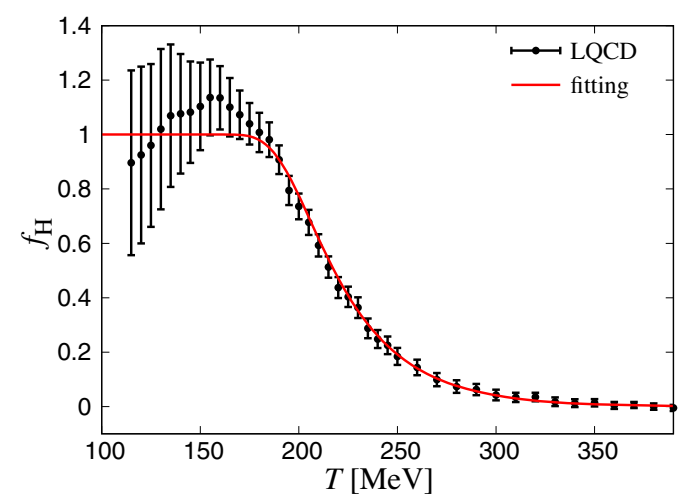

FIG. 3. $T$ dependence of the switching function $f_{\mathrm{H}}(T)$. The dots with error bars are $f_{\mathrm{H}}(T)$ of Eq. (37). The solid line is a fitting function for the $f_{\mathrm{H}}(T)$; see the text for the fitting.

$$
f_{\mathrm{H}}(T)=\frac{s_{\mathrm{LQCD}}(T)-s_{\mathrm{Q}}(T)}{s_{\text {inv }: \mathrm{H}}(T)-s_{\mathrm{Q}}(T)}
$$

In Fig. 3, $f_{\mathrm{H}}(T)$ of Eq. (37) is shown by dots with error bars. The errors come from $s_{\mathrm{LQCD}}$. The solid line is a fitting function for $f_{\mathrm{H}}(T)$ of Eq. (37); in the $\chi^{2}$ fitting, the line is assumed to be 1 in $T<180 \mathrm{MeV}$. From now on, we regard
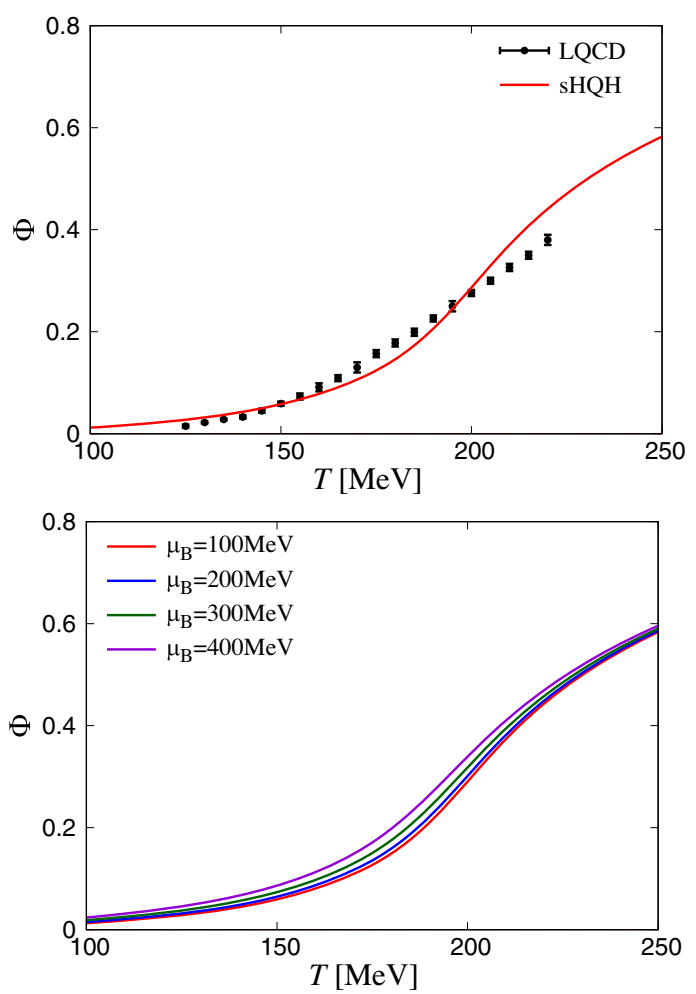

FIG. 4. $T$ dependence of the Polyakov loop $\Phi$. The upper panel is for $\mu_{B}=0 \mathrm{MeV}$, and the lower panel is for $\mu_{B}=100,200$, $300,400 \mathrm{MeV}$. The sHQH model results are shown by the solid lines. In the lower panel, four lines correspond to the cases of $\mu_{B}=100,200,300,400 \mathrm{MeV}$ from right to left. LQCD data are taken from Ref. [5].
TABLE II. Comparison between lattice transition temperature and transition region calculated with $\mathrm{sHQH}$ model for $\mu_{B}=0$.

\begin{tabular}{lc}
\hline \hline$T_{\mathrm{c}}^{\varepsilon}$ & $T_{\mathrm{c}}^{\Delta_{l, s}: \text { LQCD }}$ \\
\hline $137-204[\mathrm{MeV}]$ & $157(4)(3)[\mathrm{MeV}]$ \\
\hline \hline
\end{tabular}

the solid line as the switching function $f_{\mathrm{H}}(T)$. The weight function $f_{\mathrm{H}}(T)$ means the occupancy of hadronic matter in the total entropy, and the condition $0 \leq f_{\mathrm{H}} \leq 1$ should be satisfied.

The pressure $P$ with no vacuum contribution is obtainable from $s_{\mathrm{LQCD}}$ of Eq. (36),

$$
P\left(T, \mu_{B}\right)=\int_{0}^{T} d T^{\prime} s\left(T^{\prime}, \mu_{B}\right) .
$$

The energy density is obtained by $\varepsilon\left(T, \mu_{B}\right)=s T$ $P+\mu_{B} n$, where $n$ is the baryon-number density.

\section{NUMERICAL RESULTS}

As mentioned in Sec. I, we can consider the transition region determined from with the peak and the half-value width of $d \varepsilon\left(T, \mu_{B}\right) / d T$ as a chiral-transition region. This statement is confirmed explicitly by analyses shown in this section.

\section{A. $T$ dependence of the Polyakov loop for $\mu_{B}=0 \sim 400 \mathrm{MeV}$}

Figure 4 shows the Polyakov loop $\Phi$ as a function of $T$ for the cases of $\mu_{B}=0,100,200,300,400 \mathrm{MeV}$. The LQCD result is available only for $\mu_{B}=0 \mathrm{MeV}$ [5]. In the upper panel for $\mu_{B}=0 \mathrm{MeV}$, the $\mathrm{sHQH}$ result (solid line) well reproduces LQCD one in which the continuum limit is

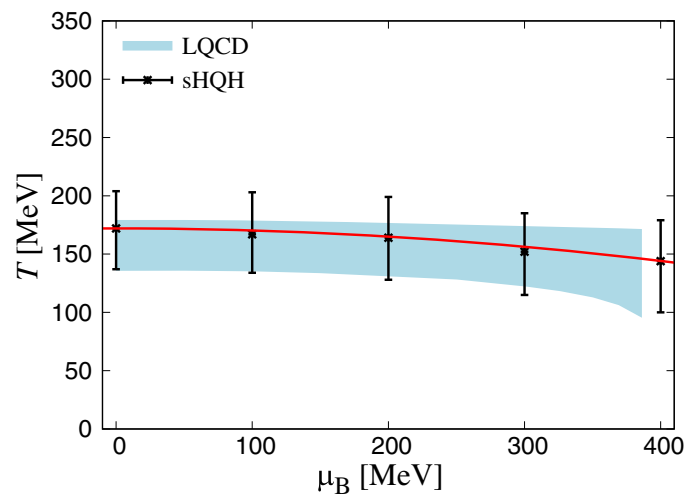

FIG. 5. Crossover region determined from $d \varepsilon / d T$ in $\mu_{B}-T$ plane. The blue band is the chiral-transition region determined by analytic continuation of LQCD simulations from imaginary to real $\mu$ [8]. The horizontal line with cross stands for the transition region determined from $d \varepsilon / d T$ and is calculated with the $\mathrm{sHQH}$ model. The transition line (red solid line), obtained by connecting the crosses, is expressed by $T=172\left(1-0.038\left(\mu_{B} / 172\right)^{2}\right) \mathrm{MeV}$. 
taken. We then predict $\Phi$ for $\mu_{B}=100,200,300,400 \mathrm{MeV}$ in the lower panel. $\mu_{B}$ dependence of $\Phi$ is small.

\section{B. Transitions}

We first consider the case of $\mu_{B}=0$. Table II shows results of $\mathrm{sHQH}$ model for the transition region $T_{\mathrm{c}}^{\varepsilon}$ determined from the peak and the half-valued width of $d \varepsilon\left(T, \mu_{B}\right) / d T$. The result is compared with LQCD data [5] on the chiral-transition temperature $T_{\mathrm{c}}^{\chi: \mathrm{LQCD}}$. Obviously, $T_{\mathrm{c}}^{\Delta_{l, s}: \mathrm{LQCD}}$ is in the region $T_{\mathrm{c}}^{e}$.

Figure 5 shows the transition region determined from the peak and the half-valued width of $d \varepsilon\left(T, \mu_{B}\right) / d T$ and the lattice chiral-transition region in $\mu_{B}-T$ plane; the former is calculated with the sHQH model and the latter is analytic continuation of LQCD simulations from imaginary to real $\mu$
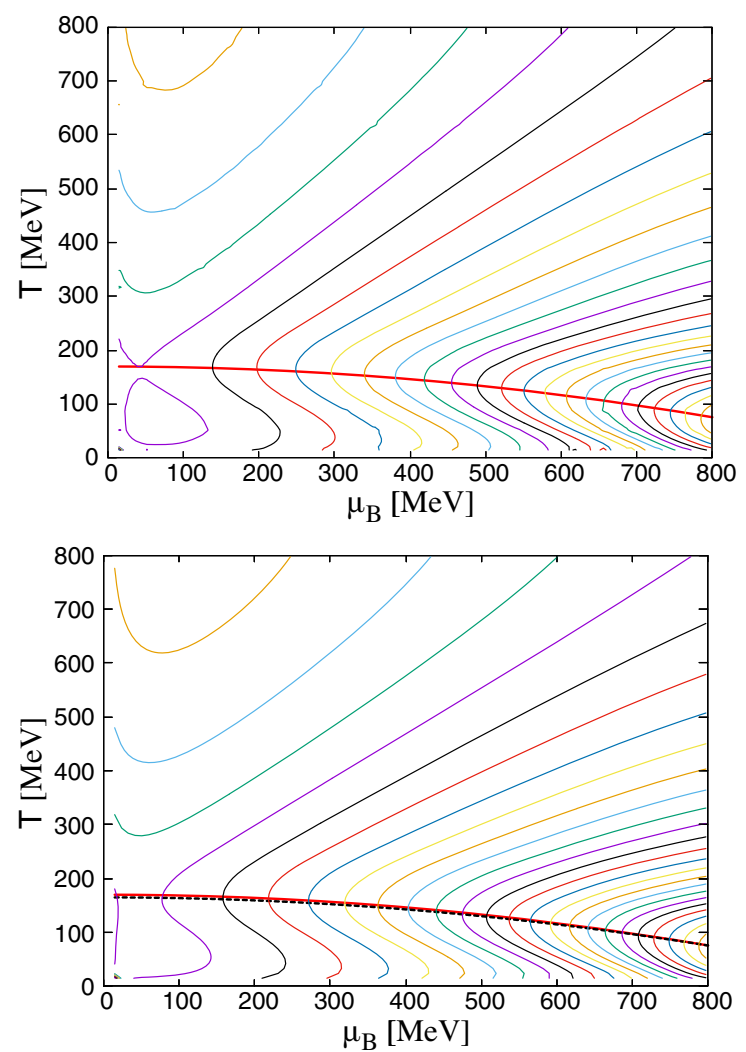

FIG. 6. Isentropic trajectories, $n / s=$ const, in $\mu_{B}-T$ plane where the strangeness neutrality is imposed for the lower panel and not for the upper panel. In the upper panel, the solid curve is a line connecting the points at which the curve of trajectory becomes maximum; the resulting curve is $T=$ $170\left(1-0.025\left(\mu_{B} / 170\right)^{2}\right) \mathrm{MeV}$. The isentropic trajectories are shown by $n / s=0.014 \sim 0.072$ from top left to bottom right. In the lower panel, the dashed line stands for a transition line with the strangeness neutrality, i.e., $T=165\left(1-0.023\left(\mu_{B} / 165\right)^{2}\right) \mathrm{MeV}$. For comparison, we also show the solid line $T=170\left(1-0.025\left(\mu_{B}\right)\right.$ $\left.170)^{2}\right) \mathrm{MeV}$ in which the strangeness neutrality is not imposed. The isentropic trajectories are shown by $n / s=0.012 \sim 0.07$ from top left to bottom right.
[8]. The transition region determined from $d \varepsilon\left(T, \mu_{B}\right) / d T$ is shown by a horizontal line with cross for each of $\mu_{B}=0$, $100,200,300,400 \mathrm{MeV}$; the cross is a maximum value of $d \varepsilon / d T$ and the horizontal line means the half-value width of $d \varepsilon / d T$. The red solid line is made by connecting the crosses. Meanwhile, the blue band indicates the width of the chiral-transition region extrapolated from the imaginary $-\mu_{B}$ region [8]. The transition region calculated with the $\mathrm{SHQH}$ model is consistent with the LQCD result. We can thus regard the transition region determined from $d \varepsilon\left(T, \mu_{B}\right) / d T$ as a chiral-crossover region.

As shown in the right panel of Fig. 4 of Ref. [35], Nonaka and Asakawa showed that in $\mu_{B}-T$ plane the isentropic trajectories are focused to the CEP. They concluded that the CEP acts as an attractor of isentropic trajectories, $n / s=$ const.

In the upper panel of Fig. 6, the solid curve is a line connecting the points at which the curvature of isentropic trajectory becomes maximum. The curve is connected to the CEP, if it exists [35]. We can thus regard the curve as a transition line in $\mu_{B}-T$ plane.

In the lower panel, we impose the strangeness neutrality. Comparing the two panels, we can find that the effect of strangeness neutrality is small. Hence, the transition calculated with $n / s$ may be deduced from relativistic nuclear collisions. There is no evidence of focusing (attractor) of isentropic trajectory in the sHQH model.

Figure 7 shows the transition line determined from $s / n$ by a solid line and the chiral-crossover region from the peak and the half-valued width of $d \varepsilon / d T$ by two dashed lines in $\mu_{B}-T$ plane. Here we do not consider the strangeness neutrality, because the effect is small. The transition line obtained from $s / n$ passes in the vicinity of dots (the peak of

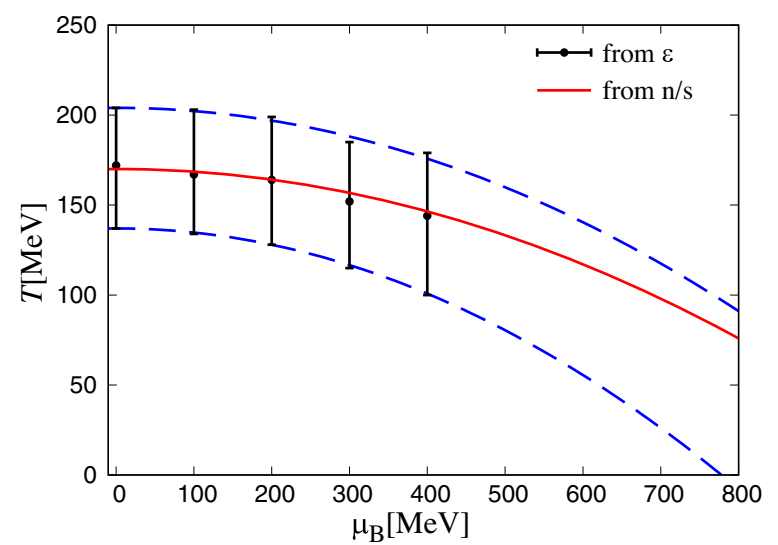

FIG. 7. Chiral-crossover region determined from $d \varepsilon / d T$ and transition line determined from $n / s$ in $\mu_{B}-T$ plane. The chiral-crossover region determined from the peak and the half-valued width of $d \varepsilon / d T$ is denoted for $\mu_{B}=0,100,200$, $300,400 \mathrm{MeV}$ by dots with error bars. The upper and lower sides of chiral-crossover region are shown by two dashed lines. The transition line determined from $n / s$ is $T=$ $170\left(1-0.025\left(\mu_{B} / 170\right)^{2}\right) \mathrm{MeV}$. 
$d \varepsilon / d T)$ and is in the chiral-crossover region. This allows us to regard the transition line determined from $s / n$ as a chiraltransition line. The quantity $s / n$ is quite useful, since it is obtainable from not ony LQCD but also heavy-ion collisions.

\section{EoS}

In this section, we do not consider the strangeness neutrality, because the effect is small.

In order to compare the present model with the previous model [20], we take the same assumption " $f_{\mathrm{H}}(T)$ has no $\mu_{B}$ dependence" in the previous model. The resulting switching function $f_{\mathrm{H}}^{\text {prev }}(T)$ is shifted to the left by about $10 \mathrm{MeV}$ from $f_{\mathrm{H}}(T)$ in Fig. 3. The difference between the present model with $f_{\mathrm{H}}(T)$ and the previous model with $f_{\mathrm{H}}^{\text {prev }}(T)$
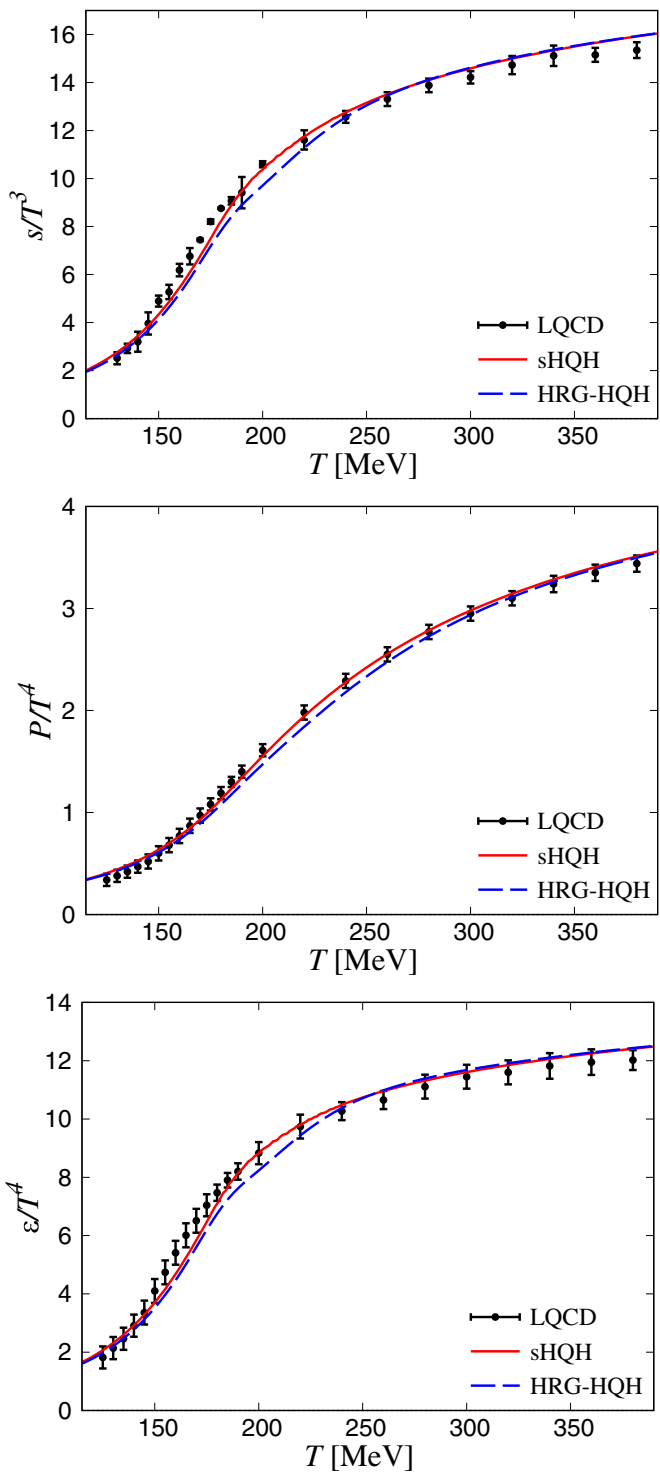

FIG. 8. $T$ dependence of $s, P, \varepsilon$ at $\mu_{B}=0 \mathrm{MeV}$. See the text for the definition of lines. LQCD data are taken from Ref. [7]. shows EV effects. The previous model with $f_{\mathrm{H}}^{\mathrm{prev}}(T)$ is referred to as "HRG-HQH model" in this paper.

Figure 8 shows $T$ dependence of $s, P, \varepsilon$, at $\mu_{B}=0 \mathrm{MeV}$. The solid and dashed lines are the results of $\mathrm{sHQH}$ and
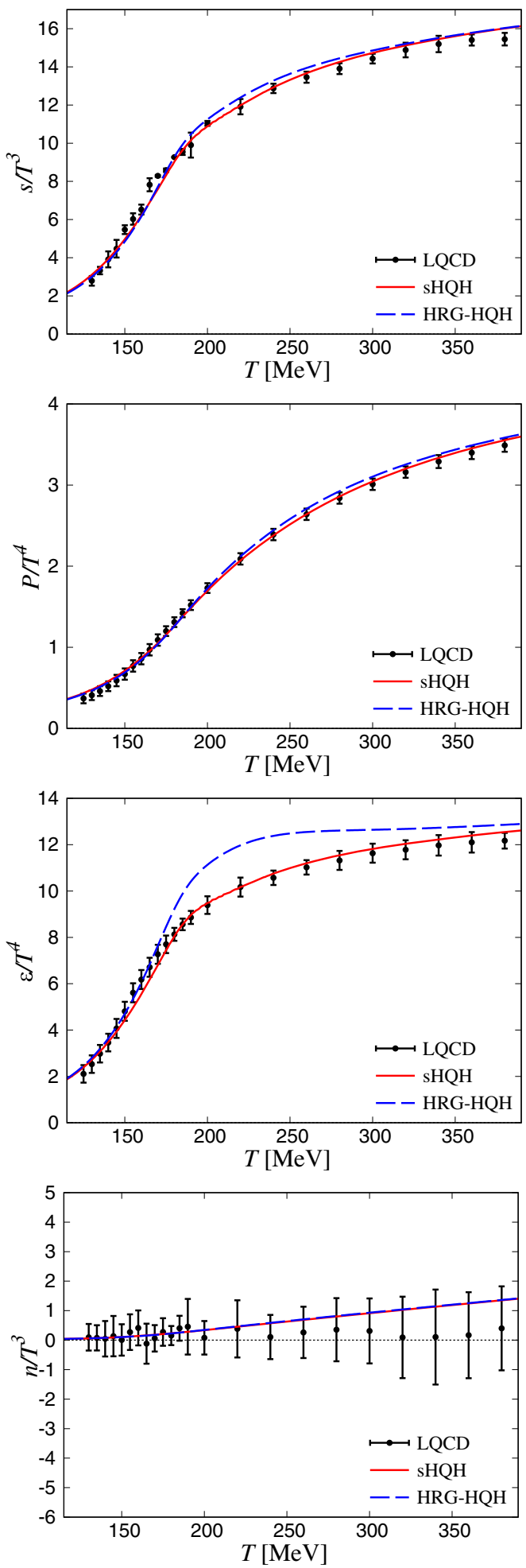

FIG. 9. $\quad T$ dependence of $s, P, \varepsilon, n$ at $\mu_{B}=200 \mathrm{MeV}$. See the text for the definition of lines. LQCD data are taken from Ref. [7]; note that $n$ is deduced from $s, P, \varepsilon$. 
HRG-HQH models, respectively. The difference between the two lines shows EV effects. We can find that the effects are small for $\mu_{B}=0 \mathrm{MeV}$. We find that the fitting of $f_{H}(T)$ is good, since the sHQH result agrees with LQCD data [7].
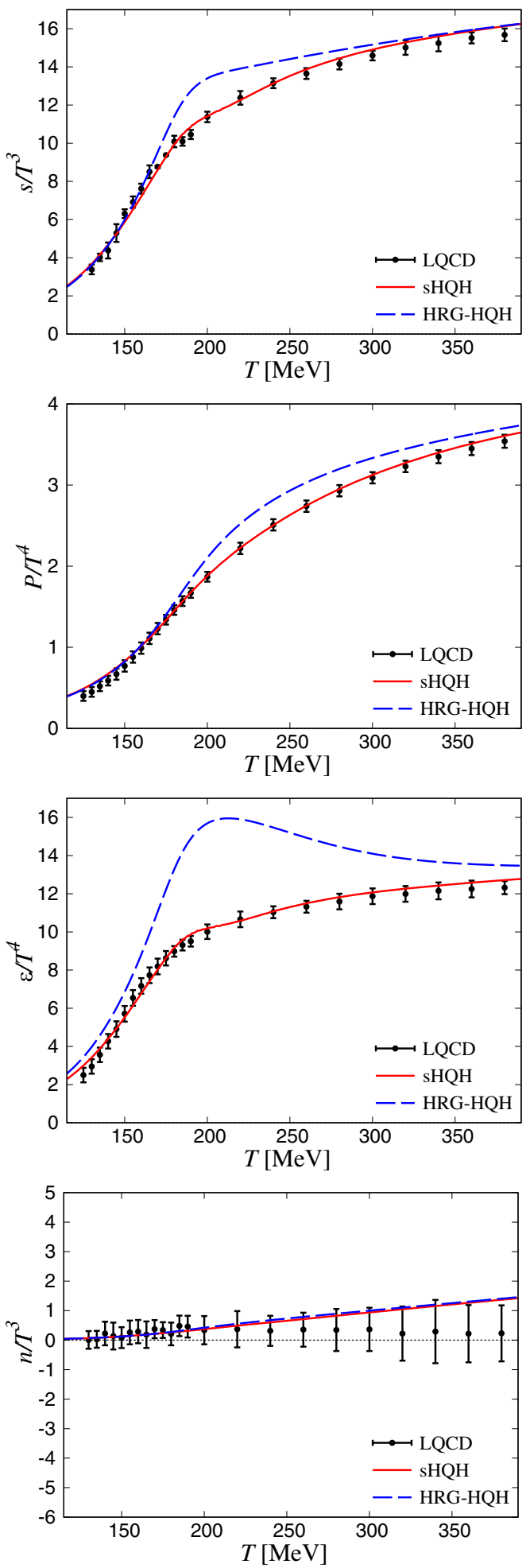

FIG. 10. $T$ dependence of $s, P, \varepsilon, n$ at $\mu_{B}=300 \mathrm{MeV}$. See the text for the definition of lines. LQCD are taken from Ref. [7]; note that $n$ is deduced from $s, P, \varepsilon$.
Also, for $P$ and $\varepsilon$, the sHQH model reproduces LQCD data.

Figures 9-11 show $T$ dependence of $s, P, \varepsilon, n$ for $\mu_{B}=200,300,400 \mathrm{MeV}$. The results of sHQH model well
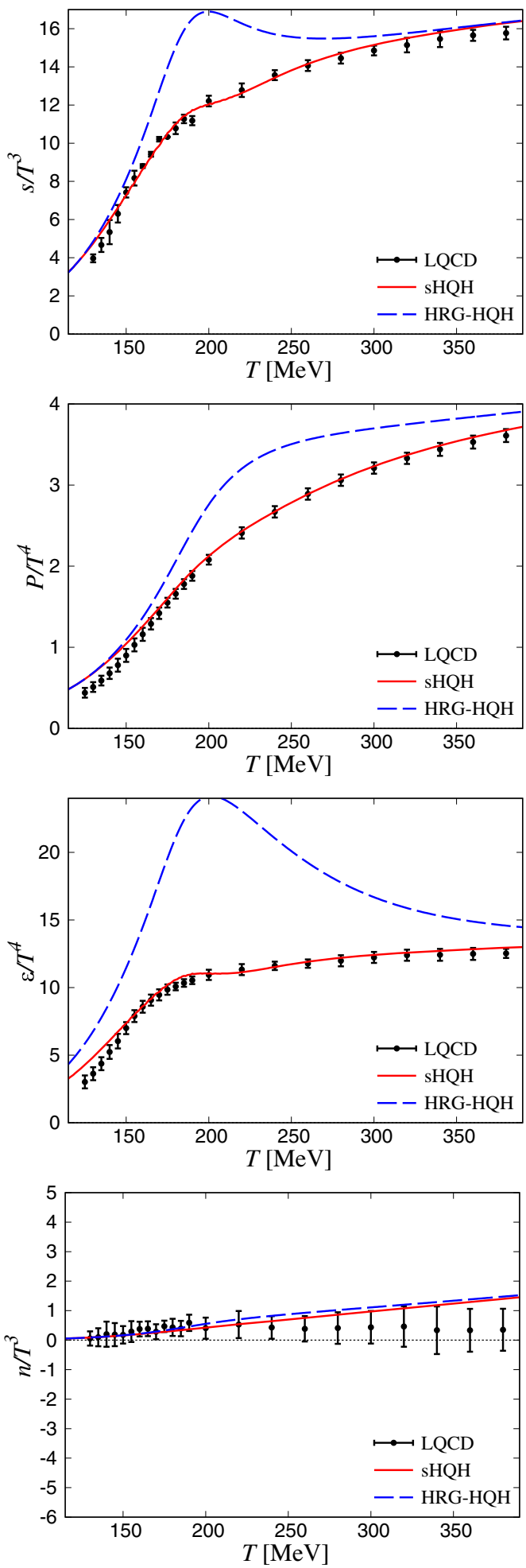

FIG. 11. $T$ dependence of $s, P, \varepsilon, n$ at $\mu_{B}=400 \mathrm{MeV}$. See the text for the definition of lines. LQCD are taken from Ref. [7]; note that $n$ is deduced from $s, P, \varepsilon$. 
reproduce the LQCD data [7]. EV effects become large as $\mu_{B}$ increases from 200 to $400 \mathrm{MeV}$. This means that the interaction between baryons becomes non-negligible as $\mu_{B}$ increases.

\section{SUMMARY}

We have constructed a simple-HQH model in the $\mu_{B}-T$ plain, improving the EV-HRG model [26,27] for the hadron piece and using the simple IQ model for the quark-gluon piece. The improved EV-HRG model yields the baryon and antibaryon pressures as simple analytic functions of Eqs. (24)-(25) and ensures that the pressure is $\mu_{B}$ even.

As an interesting result of LQCD simulations for $\mu_{B}=0$ [5], the chiral-crossover region determined from $d \Delta_{1, \mathrm{~s}} / d T$ agrees with the region from $d \varepsilon\left(T, \mu_{B}\right) / d T$. In LQCD simulations for finite $\mu_{B}$ [7], furthermore, a transition region is obtained by $d \varepsilon\left(T, \mu_{B}\right) / d T$. We may regard the transition region determined from $\varepsilon$ as a chiral-crossover region. In fact, the crossover region determined from $d \varepsilon\left(T, \mu_{B}\right) / d T$ of sHQH model agrees with the lattice result for the chiral-crossover region [8] in $\mu_{B} \leq 400 \mathrm{MeV}$. We have then predicted the chiral-crossover region in $400 \leq$ $\mu_{B} \leq 800 \mathrm{MeV}$.
In this work, we have considered that $f_{\mathrm{H}}\left(T, \mu_{B}\right)$ does not depend on $\mu_{B}$, since $s_{\text {inv: } \mathrm{H}}$ and $s_{\mathrm{Q}}$ depend on $\mu_{B}$. This allows us to determine the switching function $f_{\mathrm{H}}(T)$ from $s_{\mathrm{LQCD}}$ at $\mu_{B}=0$. The present $\mathrm{sHQH}$ with $f_{\mathrm{H}}(T)$ is successful in reproducing LQCD data on not only the chiraltransition region but also the EoS in $\mu_{B} \leq 400 \mathrm{MeV}$. In addition, the present $\mathrm{sHQH}$ model accounts for LQCD data on the Polyakov loop at $\mu_{B}=0 \mathrm{MeV}$. We have then predicted the Polyakov loop for $\mu_{B}=100,200$, 300, $400 \mathrm{MeV}$.

Using the simple-HQH model, we have also predicted a transition line derived from isentropic trajectories in $0 \leq \mu_{B} \leq 800 \mathrm{MeV}$. We found that there is no evidence of attractor of isentropic trajectories and the effect of strange neutrality is small for the transition line derived from isentropic trajectories. Further analyses of these properties seem to be important for both LQCD and relativistic nuclear collisions.

\section{ACKNOWLEDGMENTS}

The authors thank Junpei Sugano and Takehiro Hirakida for useful contributions.
[1] Y. Aoki, G. Endrödi, Z. Fodor, S. D. Katz, and K. K. Szabó, Nature (London) 443, 675 (2006).

[2] Z. Fodor and S. D. Katz, J. High Energy Phys. 04 (2004) 050 .

[3] Y. Aoki, A. Fodor, S. D. Katz, and K. K. Szabó, Phys. Lett. B 643, 46 (2006).

[4] Y. Aoki, S. Borsanyi, S. Durr, Z. Fodor, S. D. Katz, S. Krieg, and K. K. Szabo, J. High Energy Phys. 06 (2009) 088.

[5] S. Borsanyi, Z. Fodor, C. Hoelbling, S. D. Katz, S. Krieg, C. Ratti, and K. K. Szabó (Wuppertal-Budapest Collaboration), J. High Energy Phys. 09 (2010) 073.

[6] G. Endrodi, Z. Fodor, S. D. Katz, and K. K. Szabo, J. High Energy Phys. 04 (2011) 001.

[7] S. Borsanyi, G. Endrodi, Z. Fodor, S. D. Katz, S. Krieg, C. Ratti, and K. K. Szabo, J. High Energy Phys. 08 (2012) 053.

[8] R. Bellwied, S. Borsanyi, Z. Fodor, J. Gunther, S. D. Katz, C. Ratti, and K. K. Szabo, Phys. Lett. B 751, 559 (2015).

[9] A. Bazavov et al., Phys. Rev. D 95, 054504 (2017).

[10] S. Borsanyi, Z. Fodor, S. D. Katz, S. Krieg, C. Ratti, and K. K. Szabo, J. High Energy Phys. 01 (2012) 138.

[11] S. Borsanyi, Z. Fodor, C. Hoelbling, S. D. Katz, S. Krieg, and K. K. Szabo, Phys. Lett. B 730, 99 (2014).

[12] A. Bazavov et al. (HotQCD Collaboration), Phys. Rev. D 90, 094503 (2014).

[13] S. Borsanyi et al., Nature (London) 539, 69 (2016).
[14] D. U. Jungnickel and C. Wetterich, Phys. Rev. D 53, 5142 (1996).

[15] P. N. Meisinger and M. C. Ogilvie, Phys. Lett. B 379, 163 (1996).

[16] A. Dumitru and R. D. Pisarski, Phys. Rev. D 66, 096003 (2002).

[17] K. Fukushima, Phys. Lett. B 591, 277 (2004); Phys. Rev. D 77, 114028 (2008).

[18] Y. Sakai, K. Kashiwa, H. Kouno, and M. Yahiro, Phys. Rev. D 77, 051901(R) (2008); 78, 036001 (2008).

[19] A. Miyahara, Y. Torigoe, H. Kouno, and M. Yahiro, Phys. Rev. D 94, 016003 (2016).

[20] A. Miyahara, M. Ishii, H. Kouno, and M. Yahiro, Int. J. Mod. Phys. A 32, 1750205 (2017).

[21] M. Asakawa and T. Hatsuda, Phys. Rev. D 55, 4488 (1997).

[22] M. Albright, J. Kapusta, and C. Young, Phys. Rev. C 90, 024915 (2014).

[23] M. Albright, J. Kapusta, and C. Young, Phys. Rev. C 92, 044904 (2015).

[24] L. Landau and E. Lifshitz, Statistical Physics (Pergamon, New York, 1980).

[25] H. Kouno and F. Takagi, Z. Phys. C 45, 43 (1989).

[26] V. Vovchenko, D. V. Anchishkin, and M. I. Gorenstein, Phys. Rev. C 91, 024905 (2015).

[27] V. Vovchenko and H. Stocker, J. Phys. G 44, 055103 (2017). 
[28] V. Vovchenko, M. I. Gorenstein, and H. Stoecker, Phys. Rev. Lett. 118, 182301 (2017).

[29] T. Hamada and I. D. Johnston, Nucl. Phys. 34, 382 (1962).

[30] G. Aarts, Phys. Rev. Lett. 102, 131601 (2009).

[31] G. Aarts, L. Bongiovanni, E. Seiler, D. Sexty, and I.-O. Stamatescu, Eur. Phys. J. A 49, 89 (2013).
[32] D. Sexty, Phys. Lett. B 729, 108 (2014).

[33] G. Aarts, E. Seiler, D. Sexty, and I.-O. Stamatescu, Phys. Rev. D 90, 114505 (2014).

[34] K. V. Olive et al. (Particle Data Group), Chin. Phys. C 38, 090001 (2014).

[35] C. Nonaka and M. Asakawa, Phys. Rev. C 71, 044904 (2005). 